\title{
A Magnetized Local Supercluster and the Origin of the Highest Energy Cosmic Rays
}

\author{
Pasquale Blasi * and Angela V. Olinto ${ }^{\dagger}$ \\ Department of Astronomy $\&$ Astrophysics, and \\ Enrico Fermi Institute, The University of Chicago, \\ 5640 S. Ellis Av., Chicago, IL 60637
}

\begin{abstract}
A sufficiently magnetized Local Supercluster can explain the spectrum and angular distribution of ultra-high energy cosmic rays. We show that the spectrum of extragalactic cosmic rays with energies below $\sim 10^{20} \mathrm{eV}$ may be due to the diffusive propagation in the Local Supercluster with fields of $\sim 10^{-8}-10^{-7}$ Gauss. Above $\sim 10^{20} \mathrm{eV}$, cosmic rays propagate in an almost rectilinear way which is evidenced by the change in shape of the spectrum at the highest energies. The fit to the spectrum requires that at least one source be located relatively nearby at $\sim 10-15 \mathrm{Mpc}$ away from the Milky Way. We discuss the origin of magnetic fields in the Local Supercluster and the observable predictions of this model.
\end{abstract}

PACS numbers: 98.70.Sa, 96.40.De, 95.85.Sz, 98.38.Am, 98.62.-g

Submitted to Physical Review D

\section{INTRODUCTION}

The observed cosmic ray spectrum covers about 11 orders of magnitude, from about a $\mathrm{GeV}$ to a few $10^{11} \mathrm{GeV}$, with the remarkable property of being almost featureless. Up to a "knee," at $\sim 10^{15} \mathrm{eV}$, the power law spectrum is believed to be due to shock acceleration in supernova remnants [1]. Above the knee, the spectrum changes to a slightly steeper power law down to an "ankle" at $\sim 10^{19} \mathrm{eV}$ where a flattening is observed. Between the knee and the ankle, cosmic rays are considered to be produced in the Galaxy, though the mechanism is not yet clear. Above $10^{19} \mathrm{eV}$, the proton gyroradius in the Galactic magnetic field is larger than the size of the halo. This together with the lack of identified Galactic sources in the arrival direction of the events suggest an extragalactic origin for these ultra-high energy cosmic rays (UHECRs).

\footnotetext{
*e-mail: blasi@oddjob.uchicago.edu

†e-mail: olinto@oddjob.uchicago.edu
} 
If the sources of UHECRs are extragalactic, then their distances cannot be much larger than 50-100 Mpc due to the photopion production off the cosmic background radiation [2]. Protons with energies above $\sim 5 \times 10^{19} \mathrm{eV}$ experience significant losses manifested as the Greisen-Zatsepin-Kuzmin (GZK) cutoff in the spectrum. This limitation in the volume available for sources together with the extreme energies required for acceleration and the lack of source counterpart identifications make the origin of the highest energy events a challenging mystery.

Crucial for the ability to point back at possible sources is the magnetic structure of the medium between the source and us. At present, the structure of magnetic fields in the extragalactic medium is poorly known. Evidence for equipartition fields in some clusters of galaxies is well established while extragalactic fields on very large scales are constrained by Faraday rotation measures from distant quasars to magnitudes below $\sim 10^{-9}$ Gauss for a reversal scale of $1 \mathrm{Mpc}$ [3]. The deflection angle of UHECRs in extragalactic fields below this upper limit is only a few degrees for sources closer than $100 \mathrm{Mpc}$, therefore the sources should be visible in other wavelenghts within the error box of the detected events. To date, no plausible source counterparts have been found within a few degrees from the arrival direction.

Here we argue that the lack of counterpart identifications for energies below $10^{20} \mathrm{eV}$ is a natural consequence of a close to diffusive propagation in a magnetized Local Supercluster (LSC) and that counterparts may be more easily identified at energies above the limit of detectability of current experiments. In addition, we show that the transition from diffusive to straight line propagation of UHECRs in the LSC gives a good fit to the spectral changes observed with current experiments. These findings show that a simple model for UHECRs based on one or more nearby continuos sources can explain the presently available data on UHECRs if the LSC has relatively strong magnetic fields $\left(\sim 10^{-8}-10^{-7}\right.$ Gauss $)$. Such fields are allowed by current limits on extragalactic magnetic fields and could indicate the presence of magnetic fields on cosmological scales.

\section{THE LOCAL SUPERCLUSTER AND ITS MAGNETIC FIELD}

The Local Supercluster is a flattened overdensity of galaxies extending for $\sim 30-40$ $\mathrm{Mpc}$ with a width of about $\sim 10 \mathrm{Mpc}$ [1]. Our Galaxy is located at the edge of this distribution, while the Virgo cluster is approximately in the center, $\sim 17 \mathrm{Mpc}$ away from us. This large scale distribution intersects almost perpendicularly the Galactic plane defining the Supergalactic Plane and is most prominent in the northern Galactic hemisphere.

Sources of UHECRs are likely to be embedded in the LSC since it contains several active galaxies which can produce energetic particles and are close enough to survive the photopion energy losses. Thus, it would be natural to assume a correlation between the arrival direction of UHECRs and the location of the supergalactic plane. Early studies suggested a significant correlation for cosmic rays with energies $E>4 \times 10^{19} \mathrm{eV}$ 《], but further analysis using the larger AGASA data set did not find appreciable departures from an isotropic distribution in the observable region of the sky [5]. In the scenario proposed here, the correlations should only become significant for energies $E>10^{20} \mathrm{eV}$.

The magnetic field structure of the LSC is very poorly known. Direct measurements

through Faraday rotation give upper limits to fields averaged along the line of sight to 
particular background radio sources. Assuming a reversal scale of $1 \mathrm{Mpc}$, magnetic fields present over cosmological distances are constrained to have $\bar{B}_{\mathrm{Mpc}} \lesssim 10^{-9}$ Gauss, while for reversal scales close to the present horizon, $\bar{B}_{\mathrm{H}_{0}^{-1}} \lesssim 10^{-11}$ Gauss, if most of the Universe is filled with plasma. If there are significant voids in the plasma distribution in the Universe, $\bar{B}_{\mathrm{Mpc}}$ may exceed the $10^{-9}$ Gauss limit. In addition, fields with smaller reversal scales or in denser regions of the Universe can be significantly stronger than these limiting strengths. In fact, direct observations of a bridge-like structure that connects A1367 and the Coma clusters, shows the presence of magnetic fields of the order of $\mu G$ far from the clusters' core [6]. The LSC is likely to have similar overdensities to these filamentary structures and have fields that greatly exceed the $10^{-9}$ Gauss limit. In fact, Vallee [7] showed that in the region within $20^{\circ}$ around the center of the Virgo cluster, Faraday rotation measures indicate fields of about $1.5 \times 10^{-6}$ Gauss.

Superclusters are most probably not virialized structures. Therefore, equipartition magnetic fields are unlikely to be generated by the gravitational dynamics of these large scale systems alone. Extragalactic fields such as those observed around the Coma cluster are more likely to originate from local pollution by normal and radio galaxies or through the amplification of a primordial magnetic field. These two possibilities imply different structures for the present large scale magnetic fields. In particular, the structure on the largest scales and the filling factors for extragalactic fields are likely to depend on the origin of the fields.

Magnetized outflows from galaxies such as jets and winds efficiently pollute the extragalactic medium if most galaxies lived through an active phase in their history [8]. If intercluster fields are due to galactic outflows, the resulting field will be randomly oriented within cells of sizes below the mean separation between galaxies, i.e., $l_{\text {cell }} \lesssim 0.5-1 \mathrm{Mpc}$. The outflows are likely to generate turbulent fields with structure on scales below $l_{\text {cell }}$.

Primordial magnetic fields can also give rise to large scale magnetic fields. As superclusters form, primordial fields are amplified due to the density increase $\left(B \propto \rho^{2 / 3}\right)$ and due to peculiar velocities of the gravitating gas. Overdensities in superclusters vary between 1 to 100 times the critical density, therefore supercluster fields may reach $\sim 20$ times the average cosmological magnetic field on Mpc scales as the LSC starts to form. In addition, peculiar motions within the LSC may amplify a seed primordial field to $\lesssim 10^{-6}$ Gauss 11 .

Although primordial fields start with very little structure below $10 \mathrm{Mpc}$ after recombination [9], peculiar motions induced by gravitational collapse generate magnetic modes on smaller scales. Numerical simulations of structure formation indicate the development of a cascade to high wavenumbers similar to a Kolmogorov spectrum [10]. Therefore, the spectrum of magnetic fields generated by primordial fields or by outflows may not be easily distinguished on scales below the mean separation between galaxies.

As we discuss below, the energy dependence of the diffusion coefficient for UHECRs depends primarily on the strength and spectrum of the magnetic field in the LSC. Given the present observations, we consider the strength of the average magnetic field in the LSC to lie in the range between $10^{-8}-10^{-7}$ Gauss with a Kolmogorov spectrum below the largest eddy scale of $l_{\text {cell }} \lesssim 0.5-1 \mathrm{Mpc}$. 


\section{THE PROPAGATION OF COSMIC RAYS IN THE LOCAL SUPERCLUSTER}

Most recent studies of the propagation of UHECR in the intergalactic medium are based on the assumption that the magnetic field cannot be larger than $\sim 10^{-9}$ Gauss and that it is coherent on scales below $\sim 1 \mathrm{Mpc}$ [13]. In this field the typical Larmor radius is $r_{L}(E) \simeq 10^{2} \mathrm{Mpc} E_{20} / B_{-9}$, where $E_{20}$ is the energy of a particle in units of $10^{20} \mathrm{eV}$ and $B_{-9}$ is the magnetic field in units of $10^{-9}$ Gauss. The typical deflection angle from the direction of the source, located at distance $d$ can be estimated assuming that the particle makes a random walk in the magnetic field [14]:

$$
\theta(E) \simeq 3.8^{\circ}\left(\frac{d}{50 M p c}\right)^{1 / 2}\left(\frac{l_{c o h}}{1 M p c}\right)^{1 / 2}\left(\frac{E}{10^{20} e V}\right)^{-1}\left(\frac{B}{10^{-9} G}\right)
$$

Here we use the fact that the Larmor radius of the particle, $r_{L}$, is much larger than the coherence scale of the field, $l_{\text {coh }}$. Given the small angle in eq. (1), it is puzzling that the search for possible sources in the error boxes of the observed events found no candidates.

As the strength of the magnetic field increases, the Larmor radius decreases and the hypothesis of straight line propagation between scatterings, that eq. (1) relies upon, breaks down. If the LSC has fields of $10^{-8}-10^{-7}$ Gauss as we propose here, a diffusive approach is more appropriate for energies $\lesssim 10^{20} \mathrm{eV}$.

The possibility of a diffusive motion for UHECR in the LSC was previously advanced in [15] and [16]. In ref. [15], the authors assumed the Virgo cluster to be the source of UHECRs and estimated the luminosity of the source required to explain the observed fluxes above $10^{19}$ $\mathrm{eV}$. They assumed a phenomenological diffusion coefficient $D(E) \propto E^{1 / 2}$ and estimated the effect of energy losses by introducing a typical time for the losses to be compared with the propagation time scale; both treatments are improved on here. In addition, at the time of their work, estimates for Virgo magnetic fields were two orders of magnitude lower than the present observed values [7]. With Virgo magnetic fields reaching $\gtrsim 10^{-6}$ Gauss, UHECRs produced by sources inside Virgo (like M87) are unable to reach us, since the diffusion timescale becomes larger than the timescale for losses [17].

In ref. [16], the authors study the diffusive propagation in the LSC assuming a bursting source of UHECR. The resulting spectra are characterized by a pronounced cut-off due to photopion production, such that the events above $\sim 10^{20} \mathrm{eV}$ cannot be fit. The problem at the highest energies is the result of considering a diffusive propagation all the way up to the highest energies.

Here we study the propagation in two different regimes; first at lower energies where diffusion works, and then at higher energies, where particles propagate almost rectilinearly. For the diffusive propagation, we follow the analytical treatment as in ref. [16], where energy losses are taken into account in the proper way by solving the transport equation for cosmic rays:

$$
\frac{\partial n}{\partial t}-\operatorname{div}(D \nabla n)+\frac{\partial[b(E) n]}{\partial E}=q(E, \vec{r}, t)
$$

Here $n(E, r, t)$ is the density of cosmic rays with energy $E$ at a distance $r$ from the source at time $t, b(E)=d E / d t$ is the rate of energy losses, $D(E)$ is the diffusion coefficient, and $q(E, \vec{r}, t)$ is the density of particles per unit time per unit energy interval injected by the 
source. In general the source function is due to a source distribution, but here we consider a single source contribution at a time, which corresponds to taking $q(E, \vec{r}, t)=Q(E, t) \delta(\vec{r})$, where $Q(E, t) \propto E^{-\gamma}$ is the differential spectrum of the source. In general the source can be operating for a finite time $T$.

The solution to eq. (2) can be easily found by introducing the Green's function

$$
G\left(\vec{r}, E, t ; \vec{r}_{g}, E_{g}, t_{g}\right)=\frac{\delta\left(t-t_{g}-\tau\right)}{\left(4 \pi \lambda\left(E, E_{g}\right)\right)^{3 / 2}} \frac{e^{-\left(\vec{r}-\vec{r}_{g}\right)^{2} / 4 \lambda}}{b(E)}
$$

where

$$
\tau\left(E, E_{g}\right) \equiv \int_{E}^{E_{g}} d \epsilon \frac{1}{b(\epsilon)}
$$

and

$$
\lambda\left(E, E_{g}\right) \equiv \int_{E}^{E_{g}} d \epsilon \frac{D(\epsilon)}{b(\epsilon)}
$$

For a point source the general solution of eq. (2) can be written as

$$
n(E, r, t)=\int_{-\infty}^{+\infty} d t_{g} \int_{0}^{E_{\max }} Q\left(E_{g}\right) G\left(r, E, t ; r_{g}, E_{g}, t_{g}\right) d E_{g}
$$

which, after using eq. (3) above, becomes

$$
n(E, r, t)=\frac{1}{b(E)} \int_{E_{g}(E, t-T)}^{E_{g}(E, t)} d E_{g} Q\left(E_{g}\right) \frac{e^{-r^{2} / 4 \lambda\left(E, E_{g}\right)}}{\left[4 \pi \lambda\left(E, E_{g}\right)\right]^{3 / 2}} .
$$

The limits of integration $E_{g}(E, \tilde{t})$ are calculated integrating the equation $d E / d t=b(E)$ back to time $\tilde{t}$ if the detected energy is $E$. More precisely the upper limit should be written as $\min \left[E_{\max }, E_{g}(E, t)\right]$, where $E_{\max }$ is the maximum energy that can be produced in the source.

Some comments about eq. (7) are in order: i) if the source is continuous or operates on time scales larger than the typical propagation time (which is a function of energy in the diffusive approach), then this equation reduces to the well known equilibrium timeindependent solution; ii) the energy losses which are relevant in the energy range above $\sim 10^{19} \mathrm{eV}$ are due to pair production $\left(p+\gamma_{M W} \rightarrow e^{+}+e^{-}+p\right)$ off microwave (MW) background photons, and photopion production $\left(p+\gamma_{M W} \rightarrow \pi+N\right.$, where $N$ is a proton or a neutron according to the charge sign of the pion). The second channel becomes relevant above $\sim 3 \times 10^{19} \mathrm{eV}$ and determines the absorption of particles which propagate for times longer than $\sim 10^{8}$ yrs. At energies below $\sim 3 \times 10^{19} \mathrm{eV}$, the typical timescales for energy losses (due to pair production) are longer than the propagation time, so that energy losses do not play an important role. In this case eq. (7) reduces to the purely diffusive solution

$$
n(E, r)=\frac{Q(E)}{4 \pi r D(E)} \text {. }
$$


This shows that the volume suppression in the diffusive regime (without losses) goes as $1 / r$ instead of $1 / r^{2}$ and that the diffusive solution results in a spectrum $n(E) \propto E^{-(\gamma+\eta)}$, if the diffusion coefficient is $D(E) \propto E^{\eta}$.

These comments are no longer valid in the energy region where photopion production becomes relevant. Moreover, at high enough energies, the diffusion approximation breaks down and particles start to propagate in close to straight lines. The precise energy at which this transition between diffusion and straight line propagation occurs is difficult to determine without a specific model for the magnetic field in the LSC. Here we consider a transition range of energies where both the diffusive and the straight line propagation approaches are insufficient. Given a specific model for the LSC magnetic field, numerical simulations are better suited to study the spectrum in the transition regime [12]. Numerical simulations are therefore complementary to the approach given here, since the diffusion coefficient can more easily account for the structure of magnetic fields on scales below numerical resolutions.

The diffusion coefficient can be calculated by

$$
D(E)=\frac{1}{3} r_{L} c \frac{B^{2}}{\int_{1 / r_{L}}^{\infty} d k P(k)},
$$

where $P(k)$ is the magnetic field power spectrum. At energies which correspond to Larmor radii smaller than the scale of magnetic cells (i.e., $r_{L}(E) \lesssim l_{\text {cell }}$ ), we assume that the particles experience a Kolmogorov spectrum of magnetic field fluctuations, $P(k)=P_{0}\left(k / k_{0}\right)^{-5 / 3}$, where $k_{0} \sim 1 / l_{\text {cell }}$ is the small wavenumber limit for the magnetic field and $3 P_{0} k_{0} / 2=B^{2}$. The corresponding diffusion coefficient is therefore $D(E) \propto E^{1 / 3}$. For energies such that $r_{L}(E) \gtrsim l_{\text {cell }}$, the diffusion coefficient is linear in energy, $D(E) \propto E$.

The Kolmogorov spectrum can be justified, if the magnetic field in the LSC has structure similar to a cascade from the largest scales $l_{\text {cell }}$ to small scales. This may be the case if outflows from galaxies and peculiar velocities of the plasma in the LSC have experienced enough turbulent motion as in [10] and [11]. However, for the limited range of energies where UHECRs are expected to be extragalactic, the resulting spectrum is not strongly dependent on the specific choice of the power index $\eta$.

More precisely, we can write the diffusion coefficient as

$$
D(E)=D_{\text {low }}(E)=1.1 \cdot 10^{34}\left(\frac{E}{10^{19} \mathrm{eV}}\right)^{1 / 3}\left(\frac{B}{5 \cdot 10^{-8} G}\right)^{-1 / 3}\left(\frac{l_{\text {cell }}}{0.5 M p c}\right)^{2 / 3} \mathrm{~cm}^{2} / \mathrm{s}
$$

for $E \lesssim E_{c}=2.3 \cdot 10^{19}\left(\frac{l_{\text {cell }}}{0.5 M p c}\right)\left(\frac{B}{5 \cdot 10^{-8} G}\right) \mathrm{eV}$, and

$$
D(E)=D_{\text {high }}(E)=D_{\text {low }}\left(E_{c}\right)\left(\frac{E}{E_{c}}\right)
$$

for $E>E_{c}$.

We can determine the limits of the diffusive approach as follows. The diffusion time from a distance $r$ for particles with energy $E$ is given by $\tau_{\text {diff }}=r^{2} / 4 D(E)$, while the time required for the straight line propagation is just $\tau_{s}=r / c$; the condition of diffusive propagation is then $\tau_{\text {diff }} \gg \tau_{s}$, or equivalently, for fixed $r$, that $E \ll 4 \cdot 10^{20} \mathrm{eV}(r / 10 \mathrm{Mpc})$ for the fiducial values of magnetic field and coherence length as in eq. (10). This condition can also be interpreted 
as an upper limit on the diffusion coefficient: $D(E) \ll r c / 4=2.3 \cdot 10^{35}(\mathrm{r} / 10 \mathrm{Mpc}) \mathrm{cm}^{2} / \mathrm{s}$, independently of the particular energy dependence of the diffusion coefficient.

When the condition $\tau_{\text {diff }} \gg \tau_{s}$ is no longer fulfilled then the propagation regime changes and cosmic rays start moving on almost rectilinear trajectories. In this regime, we estimate the number of particles arriving from a source according with the following procedure: the particles reaching us with a fixed energy $E$ must have left the source with a generation energy $E_{g}$ which can be derived by integration of the energy loss equation $d E / d t=b(E)$ for a time given by $t=r / c+t_{d}$, where $t_{d}$ is the time delay defined as

$$
t_{d}=1.1 \times 10^{7}\left(\frac{E}{10^{20} \mathrm{eV}}\right)^{-2}\left(\frac{l_{\text {cell }}}{0.5 \mathrm{Mpc}}\right) r_{10}^{2}\left(\frac{B}{5 \cdot 10^{-8} \mathrm{G}}\right)^{2} \mathrm{yrs} .
$$

The time $t$ is thus the total minimum propagation time of particles with energy $E$ from a source at distance $r$. The flux of particles with energy $E$ is given by

$$
\Phi(E, r)=\frac{1}{4 \pi r^{2}} Q\left(E_{g}(E)\right) \frac{d E_{g}}{d E},
$$

where $Q\left(E_{g}(E)\right)$ is the number of particles with energy $E$ emitted back at time $t$ with energy $E_{g}$, per unit energy and per unit time. For the LSC redshift effects are not relevant and it can be shown that $d E_{g} / d E=b\left(E_{g}\right) / b(E)$ [18], which corresponds to ignoring evolutionary effects.

Although less probable, time delays larger than $t_{d}$ are still possible, so that a better expression for the flux should include an average on the probability distribution of time delays $P\left(t_{d}\right)$, which is given in ref. [14]. The flux thus becomes:

$$
\Phi(E, r)=\frac{1}{4 \pi r^{2}} \int_{t_{d}}^{\infty} Q\left(E_{g}\left(t_{d}\right)\right) \frac{d E_{g}}{d E} P\left(t_{d}\right) d t_{d}
$$

which however does not give results appreciably different from eq. (13).

In this paper, we assume that the source spectrum is of the form $Q(E)=K E^{-\gamma}$, with $K$ related to the total cosmic ray luminosity $L_{p}$ through $K=L_{p}(\gamma-2)$, assuming $1 \mathrm{GeV}$ as the minimum energy in the cosmic ray spectrum. An important point is that in the limit of very high energies, eqs. (13) and (14) must recover the source spectrum. In fact, for $E \gtrsim(2-3) \times 10^{20} \mathrm{eV}$ the time scale for energy losses is approximately constant, $(1 / E)(d E / d t) \simeq$ constant, so that $b(E)$ is linear in energy and $d E_{g} / d E$ turns out to be a constant. Thus in this picture a flattening of the spectrum at high energy is a straightforward consequence of the transition between the diffusion and the straight line propagation regimes.

In this section, we have identified two limits that can be used to understand the general features of the UHECR spectrum: at relatively low energies, $E \lesssim 10^{19} \mathrm{eV}$, where energy losses are not important, the flux of particles detected per unit solid angle, per unit surface, per unit energy and per unit time is is well approximated by $I(r, E)=n(E, r) c /(4 \pi) \propto$ $Q(E) / r D(E)$, where the factor of $4 \pi$ comes from the fact that diffusion makes the flux locally isotropic. The spectrum then goes as $E^{-(\gamma+\eta)}$. At very high energies the spectrum flattens to the source spectrum, and the space dilution factor is again proportional to $1 / r^{2}$. In this case, the solid angle covered by a given source decreases with energy. For a distribution of sources in the LSC, the flux per unit solid angle can be estimated by $I(E, r) \approx \Phi(E, r) /(2 \pi)$, since the LSC covers about half an hemisphere in the sky. In the following section, we find the spectrum of UHECRs for specific source parameters. 


\section{SOURCES OF UHECRS IN THE LOCAL SUPERCLUSTER}

In Figs. 1 and 2, we plot the spectrum as a function of energy for a continuous source located between 10 and $17 \mathrm{Mpc}$ away from us. We vary the LSC average magnetic field between $5 \times 10^{-8}$ and $10^{-7}$ Gauss. The required luminosity for the source varies according to the spectral index, such that for $\eta=2.1, L_{p} \simeq 10^{43} \mathrm{erg} / \mathrm{s}$ is sufficient while for $\eta=2.4$, $L_{p} \gtrsim 10^{45} \mathrm{erg} / \mathrm{s}$. In Fig. 1, we assume that the maximum energy reached at the source is $E_{\max }=10^{21} \mathrm{eV}$, while in Fig. 2, $E_{\max }=10^{22} \mathrm{eV}$.

Contrasting our calculations with the data from ref. [19], we see that the solid and dotted lines can reproduce the spectrum reasonably well within the observed uncertainties. The flattening of the spectrum at higher energies is clear and the flux level is compatible with the data. These two cases correspond to sources at $13 \mathrm{Mpc}$ (solid) and $10 \mathrm{Mpc}$ (dotted) with fields between 5 and $10 \times 10^{-8}$ Gauss, respectively.

These choices for the magnetic field of the LSC and distance to the source give good fits for the following reason. The diffusive regime is a good approximation for energies below $E_{0}=8 \times 10^{19} B_{-8} r_{10} \mathrm{eV}$, for $l_{\text {cell }} \simeq 0.5 \mathrm{Mpc}$. Therefore, if we want to reproduce the flattening of the spectrum at $\sim 1-3 \times 10^{20} \mathrm{eV}$, then we need $B_{-8} r_{10}$ of a few. For magnetic fields in the range that we propose here, $B_{-8} \sim 5-10$, the sources have to be relatively nearby at about $10 \mathrm{Mpc}$. Increasing the distance to the source causes the flattening point to move to higher energies and the flux of cosmic rays to decrease drastically, with the appearance of a pronounced cut-off. In other words, a magnetized LSC brings the GZK cutoff to much shorter distances.

In addition, bursting sources cannot fit the spectrum if the LSC has fields as strong as we suggest here. The need to fit both the energies below the GZK cutoff as well as those above, constrains the sources to be continuous over a long enough period ( $\gtrsim$ Gyr) for the observed flux to reach the a steady-state signature.

If UHECRs are accelerated in active galaxies, there are a number of possible nearby sources within a $20 \mathrm{Mpc}$ radius of our Galaxy. For example, NGC 3031, M82, NGC 3115, and Cen A (NGC 5128) are all below 4 Mpcs away; NGC 4051 is about $10 \mathrm{Mpc}$ away; and NGC 4151, NGC 3227, NGC 1068, NGC 1566, and M87 are between 15 and 18 Mpc away. If the source is very close, than the deflection angles become small at the highest energies, and a counterpart to the source should be identifiable. The stronger the LSC field, the larger the typical deflection angle for the sources. Numerical simulations indicate that the deflection angle can be of the order of $10^{\circ}$ for our choices of parameters [12].

\section{CONCLUSION}

In conclusion, we have shown that a sufficiently magnetized Local Supercluster can explain the spectrum of ultra-high energy cosmic rays. The spectrum of extragalactic cosmic rays with energies below $\sim 10^{19} \mathrm{eV}$ may be due to the diffusive propagation in the Local Supercluster with fields of $\sim 5-10 \times 10^{-8}$ Gauss. Above $\sim 10^{20} \mathrm{eV}$, cosmic rays propagate in an almost rectilinear way which is evidenced by the change in shape of the spectrum at the highest energies. The transition from diffusive to straight line propagation of UHECRs in the LSC gives a good fit to the spectral changes observed with current experiments as long as at least one source be located relatively nearby at $\sim 10 \mathrm{Mpc}$ away from our Galaxy. 
Future studies of the magnetic field structure of the LSC as well as the detection of UHECRs at the highest energies will help determine the sources of UHECRs. Another consequence of our model is that the sources are relatively nearby, thus, the precise structure at the edge of the LSC is not as important as the "local" 10 to 15 Mpc radius around us. Finally, we expect a significant North-South asymmetry in the UHECR data at the highest energies, as the LSC is more prominent in the Northern Hemisphere. This asymmetry could be detected by future experiments that cover both hemispheres such as the proposed Auger Project [20].

In addition, with respect to the angular distribution of the observed events, the lack of counterpart identifications for energies below $10^{20} \mathrm{eV}$ is a natural consequence of a close to diffusive propagation in the LSC. We predict that counterparts will be more easily identified at energies above the limit of detectability of current experiments.

These findings show that a simple model for UHECRs based on one or more nearby continuos sources can explain the presently available data on UHECRs, if the LSC has relatively strong magnetic fields $\left(\sim 10^{-8}-10^{-7}\right.$ Gauss $)$. Such fields are allowed by current limits on extragalactic magnetic fields and could indicate the presence of magnetic fields on cosmological scales.

\section{ACKNOWLEDGMENTS}

We gratefully acknowledge many illuminating discussions with G. Sigl, M. Lemoine, V.S. Berezinskii, R. Rosner, P. Kronberg, and P. Biermann. P. B. was supported by a Istituto

Nazionale di Fisica Nucleare fellowship at the University of Chicago. A. V. O. was supported in part by the DOE through grant DE-FG0291 ER40606, and by the NSF through grant AST-94-20759. 


\section{REFERENCES}

[1] V. S. Berezinskii, S. V. Bulanov, V. A. Dogiel, and V. L. Ginzburg and V. S. Ptuskin, Astrophysics of Cosmic Rays ed. North-Holland, (1990).

[2] K. Greisen, Phys. Rev. Lett. 16, 748 (1966); G. T. Zatsepin and V. A. Kuzmin, Sov. Phys.-JETP Lett., 4, 78 (1966).

[3] P. P. Kronberg, Rep. Prog. Phys. 57, 325 (1994).

[4] T. Stanev, P. Biermann, J. Lloyd-Evans, J. Rachen, and A. Watson, Phys.Rev.Lett. 75, 3056 (1995).

[5] M. Nagano in the Proceedings of the XIXth Texas Symposium on Relativistic Astrophysics, ed. A. V. Olinto, J. Frieman, and D.N. Schramm, (World Scientific, Singapure, 1998).

[6] K.-T. Kim, P.C. Tribble, and P.P. Kronberg, Astrophys. J. 379, 80 (1991).

[7] J. P. Vallee, Astrophys. J. 99, 459 (1990); and M.N.R.A.S. 264, 665 (1993).

[8] P. Kronberg, and H. Lesch, in The Physics of Galactic Halos eds. H. Lesch, R-J Dettmar, U. Mebold and R. Schlickeiser, (Akademie Verlag, Berlin, 1996).

[9] K. Jedamzik, V. Katalinic, and A. V. Olinto, Phys. Rev. D 57, 3264 (1998).

[10] R. Kulsrud, D. Ryu, R. Cen, and J. P. Ostriker, Astrophys J. (1997).

[11] D. Ryu, H. Kang, and P. Biermann, e-print astro-ph/9803275.

[12] G. Sigl, M. Lemoine, and P. Bierman, in preparation.

[13] M. Lemoine, A. V. Olinto, G. Sigl, and D. Schramm, Astrophys. J. Lett. 486, L115 (1997).

[14] E. Waxman and J. Miralda-Escudè, Astrophys J. Lett. 472, L89 (1996).

[15] J. Wdowczyk and A. W. Wofendale, Nature 281, 356 (1979).

[16] V.S. Berezinsky, S.I. Grigorieva, and V.A. Dogiel Astronomy \& Astrophysics 232, 582 (1990).

[17] P. Blasi, in preparation.

[18] V.S. Berezinsky and S.I. Grigorieva, Astron and Astrophys. 199, 1 (1988).

[19] N. Hayashida, Phys. Rev. Lett. 73, 3491 (1994); D. J. Bird et al, Astrophys J. 441, 144 (1995).

[20] J. W. Cronin, in The Pierre Auger Observatory Design Report (1997). 


\section{FIGURES}

FIG. 1. Flux versus Energy assuming a maximum energy at the source of $E_{\max }=10^{21} \mathrm{eV}$. The solid line corresponds to $r=13 \mathrm{Mpc}, B=5 \times 10^{-8}$ Gauss, $\gamma=2.1$, and $L_{p}=2.2 \times 10^{43}$ $\mathrm{erg} / \mathrm{s}$; dotted line is for $r=10 \mathrm{Mpc}, B=10^{-7}$ Gauss, $\gamma=2.1$, and $L_{p}=10^{43} \mathrm{erg} / \mathrm{s}$; dashed line is for $r=10 \mathrm{Mpc}, B=10^{-7}$ Gauss, $\gamma=2.4$, and $L_{p}=3.2 \times 10^{45} \mathrm{erg} / \mathrm{s}$; and dasheddotted line corresponds to $r=17 \mathrm{Mpc}, B=5 \times 10^{-8}$ Gauss, $\gamma=2.1$, and $L_{p}=3.3 \times 10^{43}$ $\mathrm{erg} / \mathrm{s}$. The data is from ref. [19].

FIG. 2. Flux versus Energy assuming a maximum energy at the source of $E_{\max }=10^{22} \mathrm{eV}$. The solid line corresponds to $r=13 \mathrm{Mpc}, B=5 \times 10^{-8}$ Gauss, $\gamma=2.1$, and $L_{p}=2.2 \times 10^{43}$ $\mathrm{erg} / \mathrm{s}$; dotted line is for $r=10 \mathrm{Mpc}, B=10^{-7}$ Gauss, $\gamma=2.1$, and $L_{p}=10^{43} \mathrm{erg} / \mathrm{s}$; dashed line is for $r=10 \mathrm{Mpc}, B=10^{-7}$ Gauss, $\gamma=2.4$, and $L_{p}=3.2 \times 10^{45} \mathrm{erg} / \mathrm{s}$; and dasheddotted line corresponds to $r=17 \mathrm{Mpc}, B=5 \times 10^{-8}$ Gauss, $\gamma=2.1$, and $L_{p}=3.3 \times 10^{43}$ $\mathrm{erg} / \mathrm{s}$. The data is from ref. [19]. 


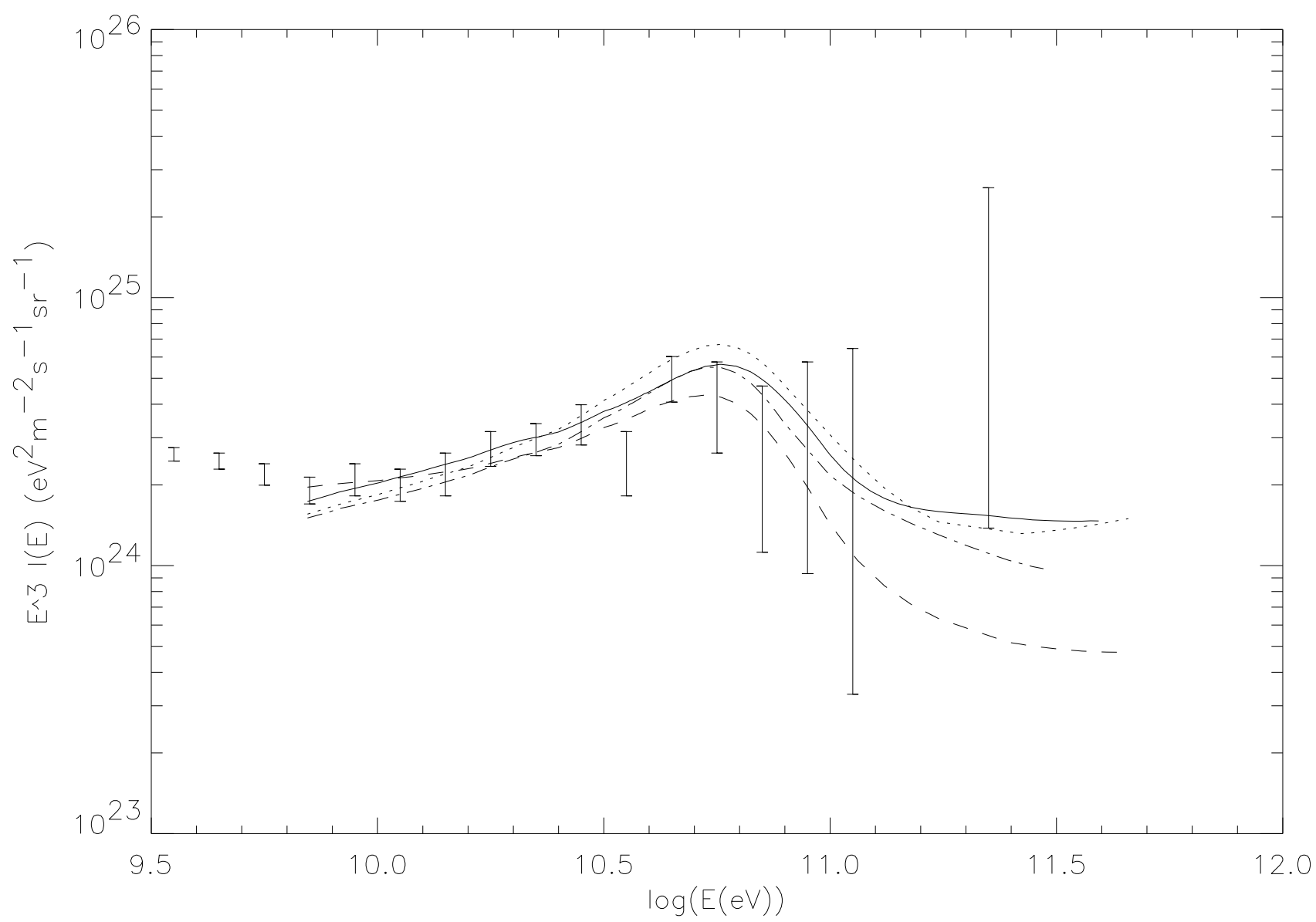




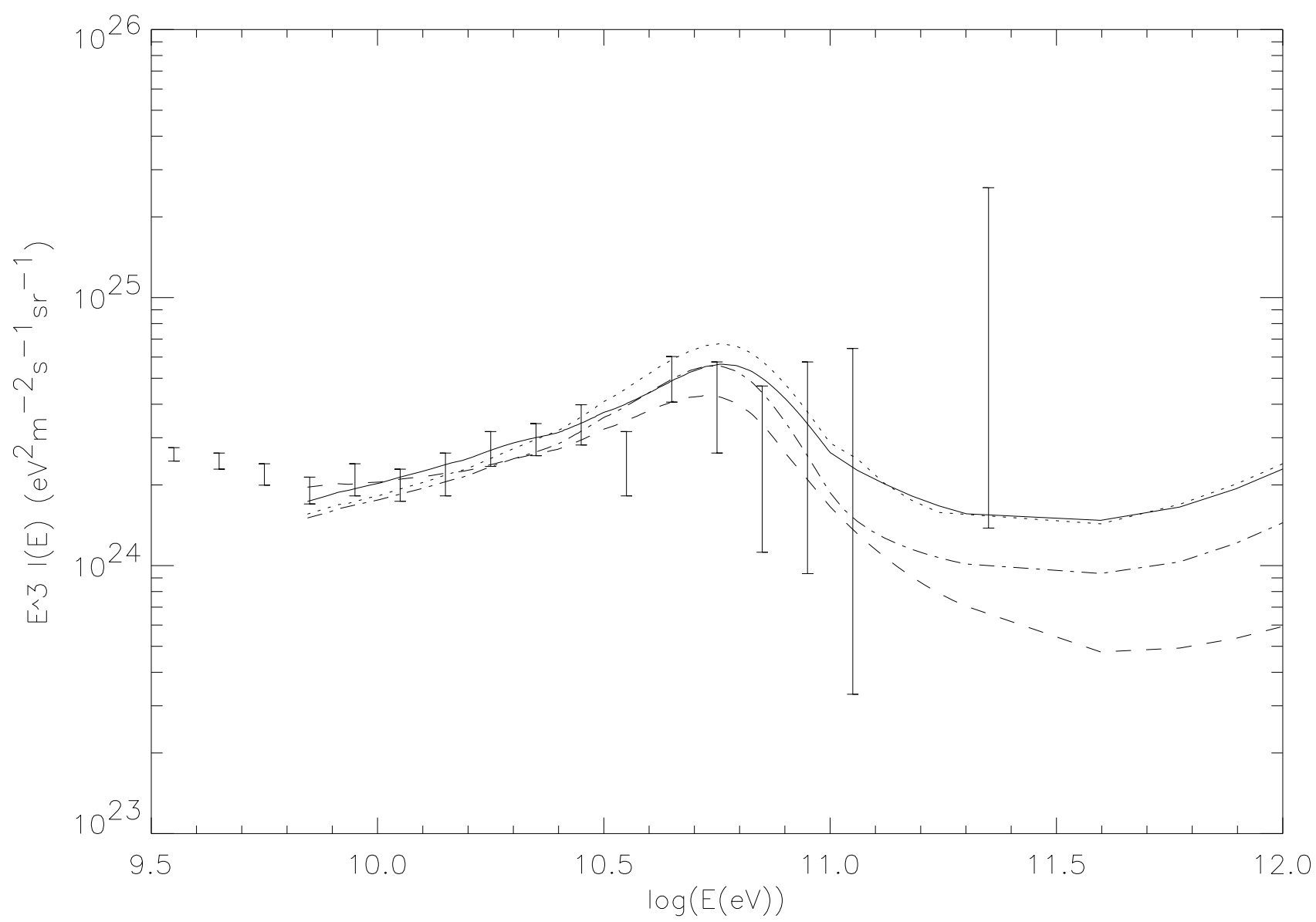

